\title{
Some Suzuki-type fixed point theorems for generalized multivalued mappings and applications
}

Dragan Đorić ${ }^{*}$ and Rade Lazović

* Correspondence: djoricd@fon.bg. ac.rs

Department of Mathematics, Faculty of Organizational Sciences, University of Belgrade, 11000 Beograd, Jove llića 154, Serbia

\section{Abstract}

In this article we obtain a Suzuki-type generalization of a fixed point theorem for generalized multivalued mappings of Ćirić (Matematićki Vesnik, 9(24), 265-272, 1972). The obtained results extend furthermore the recently developed Kikkawa-Suzuki-type contractions. Applications to certain functional equations arising in dynamic programming are also considered.

Keywords: Complete metric space, fixed point, multivalued mapping, functional equation

\section{Introduction and preliminaries}

In 2008 Suzuki [1] introduced a new type of mappings which generalize the wellknown Banach contraction principle [2]. Some others [3] generalized Kannan mappings [4].

Theorem 1.1. (Kikkawa and Suzuki [3]) Let T be a mapping on complete metric space $(X, d)$ and let $\phi$ be a non-increasing function from $[0,1)$ into $(1 / 2,1]$ defined by

$$
\varphi(r)= \begin{cases}1, & \text { if } 0 \leq r \leq \frac{1}{\sqrt{2}} \\ \frac{1}{1+r}, & \text { if } \frac{1}{\sqrt{2}} \leq r<1 .\end{cases}
$$

Let $\alpha \in[0,1 / 2)$ and $r=\alpha /(1-\alpha) \in[0,1)$. Suppose that

$$
\varphi(r) d(x, T x) \leq d(x, y) \text { implies } d(T x, T y) \leq \alpha d(x, T x)+\alpha d(y, T y)
$$

for all $x, y \in X$. Then, $T$ has a unique fixed point $z$, and $\lim _{n} T^{n} x=z$ holds for every $x \in X$.

Theorem 1.2. (Kikkawa and Suzuki [3]) Let $T$ be a mapping on complete metric space $(X, d)$ and $\theta$ be a nonincreasing function from $[0,1)$ onto $(1 / 2,1]$ defined by

$$
\theta(r)= \begin{cases}1 \quad \text { if } 0 \leq r \leq \frac{1}{2}(\sqrt{5}-1), \\ \frac{1-r}{r^{2}} \text { if } \frac{1}{2}(\sqrt{5}-1) \leq r \leq \frac{1}{\sqrt{2}} \\ \frac{1}{1+r} \text { if } \frac{1}{\sqrt{2}} \leq r<1 .\end{cases}
$$

(c) 2011 Đorićć and Lazovićć; licensee Springer. This is an Open Access article distributed under the terms of the Creative Commons Attribution License (http://creativecommons.org/licenses/by/2.0), which permits unrestricted use, distribution, and reproduction in any medium, provided the original work is properly cited. 
Suppose that there exists $r \in[0,1)$ such that

$$
\theta(r) d(x, T x) \leq d(x, y) \text { implies } d(T x, T y) \leq r \max \{d(x, T x), d(y, T y)\}
$$

for all $x, y \in X$. Then, $T$ has a unique fixed point $z$, and $\lim _{n} T^{n} x=z$ holds for every $x \in X$.

On the other hand, Nadler [5] proved multivalued extension of the Banach contraction theorem.

Theorem 1.3. (Nadler [5]) Let $(X, d)$ be a complete metric space and let $T$ be a mapping from $X$ into $C B(X)$. Assume that there exists $r \in[0,1)$ such that

$$
H(T x, T y) \leq r d(x, y)
$$

for all $x, y \in X$. Then, there exists $z \in X$ such that $z \in T z$.

Many fixed point theorems have been proved by various authors as generalizations of the Nadler's theorem (see [6-9]). One of the general fixed point theorems for a generalized multivalued mappings appears in [10].

The following result is a generalization of Nadler [5].

Theorem 1.4. (Kikkawa and Suzuki [11]) Let $(X, d)$ be a complete metric space, and let $T$ be a mapping from $X$ into $C B(X)$. Define a strictly decreasing function $\eta$ from $[0$, 1) onto $(1 / 2,1]$ by

$$
\eta(r)=\frac{1}{1+r}
$$

and assume that there exists $r \in[0,1)$ such that

$$
\eta(r) d(x, T x) \leq d(x, y) \text { implies } H(T x, T y) \leq r d(x, y)
$$

for all $x, y \in X$. Then, there exists $z \in X$ such that $z \in T z$.

In this article we obtain a Kikkawa-Suzuki-type fixed point theorem for generalized multivalued mappings considered in [10]. The result obtained here complement and extend some previous theorems about multivalued contractions. In addition, using our result, we proved the existence and uniqueness of solutions for certain class of functional equations arising in dynamic programming.

\section{Main results}

Let $(X, d)$ be a metric space. We denote by $C B(X)$ the family of all nonempty, closed, bounded subsets of $X$. Let $H(\cdot, \cdot)$ be the Hausdorff metric, that is,

$$
H(A, B)=\max \left\{\sup _{a \in A} d(a, B), \sup _{b \in B} d(A, b)\right\}
$$

for $A, B \in C B(X)$, where $d(x, B)=\inf _{y \in B} d(x, y)$.

Now, we will prove our main result.

Theorem 2.1. Define a nonincreasing function $\phi$ from $[0,1)$ into $(0,1]$ by

$$
\varphi(r)=\left\{\begin{array}{l}
1, \quad \text { if } 0 \leq r<\frac{1}{2} \\
1-r, \text { if } \frac{1}{2} \leq r<1 .
\end{array}\right.
$$

Let $(X, d)$ be a complete metric space and $T$ be a mapping from $X$ into $C B(X)$. Assume that there exists $r \in[0,1)$ such that $\phi(r) d(x, T x) \leq d(x, y)$ implies 


$$
H(T x, T y) \leq r \cdot \max \left\{d(x, y), d(x, T x), d(y, T y), \frac{d(x, T y)+d(y, T x)}{2}\right\}
$$

for all $x, y \in X$. Then, there exists $z \in X$ such that $z \in T z$.

Proof.

1. Let $r_{1}$ be such a real number that $0 \leq r<r_{1}<1$, and $u_{1} \in X$ and $u_{2} \in T u_{1}$ be arbitrary. Since $u_{2} \in T u_{1}$, then $d\left(u_{2}, T u_{2}\right) \leq H\left(T u_{1}, T u_{2}\right)$ and, as $\phi(r)<1$,

$$
\varphi(r) d\left(u_{1}, T u_{1}\right) \leq d\left(u_{1}, T u_{1}\right) \leq d\left(u_{1}, u_{2}\right) .
$$

Thus, from the assumption (3),we have

$$
\begin{aligned}
d\left(u_{2}, T u_{2}\right) & \leq H\left(T u_{1}, T u_{2}\right) \\
& \leq r \cdot \max \left\{d\left(u_{1}, u_{2}\right), d\left(u_{1}, T u_{1}\right), d\left(u_{2}, T u_{2}\right), \frac{d\left(u_{1}, T u_{2}\right)+0}{2}\right\} \\
& \leq r \cdot \max \left\{d\left(u_{1}, u_{2}\right), d\left(u_{2}, T u_{2}\right), \frac{d\left(u_{1}, u_{2}\right)+d\left(u_{2}, T u_{2}\right)}{2}\right\} .
\end{aligned}
$$

Hence, as $r<1$, we have $d\left(u_{2}, T u_{2}\right) \leq r d\left(u_{1}, u_{2}\right)$. Hence, there exists $u_{3} \in T u_{2}$ such that $d\left(u_{2}, u_{3}\right) \leq r_{1} d\left(u_{1}, u_{2}\right)$. Thus, we can construct such a sequence $\left\{u_{n}\right\}$ in $X$ that

$$
u_{n+1} \in T u_{n} \text { and } d\left(u_{n+1}, u_{n+2}\right) \leq r_{1} d\left(u_{n}, u_{n+1}\right) .
$$

Then, we have

$$
\sum_{n=1}^{\infty} d\left(u_{n}, u_{n+1}\right) \leq \sum_{n=1}^{\infty} r_{1}^{n-1} d\left(u_{1}, u_{2}\right)<\infty
$$

Hence, we conclude that $\left\{u_{n}\right\}$ is a Cauchy sequence. Since $X$ is complete, there is some point $z \in X$ such that

$$
\lim _{n \rightarrow \infty} u_{n}=z
$$

2. Now, we will show that

$$
d(z, T x) \leq r \cdot \max \{d(z, x), d(x, T x)\} \text { for all } x \in X \backslash\{z\} .
$$

Since $u_{n} \rightarrow z$, there exists $n_{0} \in N$ such that $d\left(z, u_{n}\right) \leq(1 / 3) d(z, x)$ for all $n \geq n_{0}$. Then, we have

$$
\begin{aligned}
\varphi(r) d\left(u_{n}, T u_{n}\right) & \leq d\left(u_{n}, T u_{n}\right) \\
& \leq d\left(u_{n}, u_{n+1}\right) \\
& \leq d\left(u_{n}, z\right)+d\left(u_{n+1}, z\right) \\
& \leq \frac{2}{3} d(x, z) .
\end{aligned}
$$

Thus,

$$
\varphi(r) d\left(u_{n}, T u_{n}\right) \leq \frac{2}{3} d(x, z)
$$


Since

$$
\begin{aligned}
\frac{2}{3} d(x, z) & =d(x, z)-\frac{1}{3} d(x, z) \\
& \leq d(x, z)-d\left(u_{n}, z\right) \\
& \leq d\left(u_{n}, x\right)
\end{aligned}
$$

from (5), we have $\phi(r) d\left(u_{n}, T u_{n}\right) \leq d\left(u_{n}, x\right)$. Then, from (3),

$$
\begin{gathered}
H\left(T u_{n}, T x\right) \leq r \cdot \max \left\{d\left(u_{n}, x\right), d\left(u_{n}, T u_{n}\right), d(x, T x),\right. \\
\left.\frac{d\left(u_{n}, T x\right)+d\left(x, T u_{n}\right)}{2}\right\} .
\end{gathered}
$$

Since $u_{n+1} \in T u_{n}$, then

$$
d\left(u_{n+1}, T x\right) \leq H\left(T u_{n}, T x\right) \text { and } d\left(u_{n}, T u_{n}\right) \leq d\left(u_{n}, u_{n+1}\right) .
$$

Hence, from (6), we get

$$
d\left(u_{n+1}, T x\right) \leq r \cdot \max \left\{d\left(u_{n}, x\right), d\left(u_{n}, u_{n+1}\right), d(x, T x), \frac{d\left(u_{n}, T x\right)+d\left(x, u_{n+1}\right)}{2}\right\}
$$

for all $n \in N$ with $n \geq n_{0}$. Letting $n$ tend to $\infty$, we obtain (4).

3. Now, we will show that $z \in T z$.

3.1. First, we consider the case $0 \leq r<\frac{1}{2}$. Suppose, on the contrary, that $z \notin T z$. Let $a \in T z$ be such that $2 r d(a, z)<d(z, T z)$. Since $a \in T z$ implies $a \neq z$, then from (4) we have

$$
d(z, T a) \leq r \max \{d(z, a), d(a, T a)\}
$$

On the other hand, since $\phi(r) d(z, T z) \leq d(z, T z) \leq d(z, a)$, then from (3) we have

$$
\begin{aligned}
H(T z, T a) & \leq r \cdot \max \left\{d(z, a), d(z, T z), d(a, T a), \frac{d(z, T a)+0}{2}\right\} \\
& \leq r \max \{d(z, a), d(z, T z), d(a, T a)\} \\
& \leq r \max \{d(z, a), d(a, T a)\} .
\end{aligned}
$$

Hence,

$$
d(a, T a) \leq H(T z, T a) \leq r \max \{d(z, a), d(a, T a)\} .
$$

Hence, $d(a, T a) \leq r d(z, a)<d(z, a)$, and from (7), we have $d(z, T a) \leq r d(z, a)$. Therefore, we obtain

$$
\begin{aligned}
d(z, T z) & \leq d(z, T a)+H(T a, T z) \\
& \leq d(z, T a)+r \max \{d(z, a), d(a, T a)\} \\
& \leq 2 r d(z, a) \\
& <d(z, T z) .
\end{aligned}
$$

This is a contradiction. As a result, we have $z \in T z$.

3.2. Now, we consider the case $\frac{1}{2} \leq r<1$. We will first prove

$$
H(T x, T z) \leq r \max \left\{d(x, z), d(x, T x), d(z, T z), \frac{d(, T x)+d(z, T x)}{2}\right\}
$$


for all $x \in X$. If $x=z$, then the previous obviously holds. Hence, let us assume $x \neq z$. Then, for every $n \in N$, there exists a sequence $y_{n} \in T x$ such that $d\left(z, y_{n}\right) \leq d(z, T x)+$ $(1 / n) d(x, z)$. Using (4), we have for all $n \in N$

$$
\begin{aligned}
d(x, T x) & \leq d\left(x, y_{n}\right) \\
& \leq d(x, z)+d\left(z, y_{n}\right) \\
& \leq d(x, z)+d(z, T x)+\frac{1}{n} d(x, z) \\
& \leq d(x, z)+r \max \{d(x, z), d(x, T x)\}+\frac{1}{n} d(x, z) .
\end{aligned}
$$

If $d(x, z) \geq d(x, T x)$, then

$$
d(x, T x) \leq d(x, z)+r d(x, z)+\frac{1}{n} d(x, z)=\left(1+r+\frac{1}{n}\right) d(x, z) .
$$

Letting $n$ tend to $\infty$, we have $d(x, T x) \leq(r+1) d(x, z)$. Thus,

$$
\varphi(r) d(x, T x)=(1-r) d(x, T x) \leq \frac{1}{r+1} d(x, T x) \leq d(x, z)
$$

and from (3), we have (8).

If $d(x, z)<d(x, T x)$, then

$$
d(x, T x) \leq d(x, z)+r d(x, T x)+\frac{1}{n} d(x, z)
$$

and therefore,

$$
(1-r) d(x, T x) \leq\left(1+\frac{1}{n}\right) d(x, z)
$$

Letting $n$ tend to $\infty$, we have $\phi(r) d(x, T) \leq d(x, z)$ and thus, from (3), we again have (8).

Finally, from (8), we obtain

$$
\begin{aligned}
d(z, T z) & =\lim _{n \rightarrow \infty} d\left(u_{n+1}, T z\right) \\
& \leq \lim _{n \rightarrow \infty} r \max \left\{d\left(u_{n}, z\right), d\left(u_{n}, T u_{n}\right), d(z, T z), \frac{d\left(u_{n}, T z\right)+d\left(z, T u_{n}\right)}{2}\right\} \\
& \leq \lim _{n \rightarrow \infty} r \max \left\{d\left(u_{n}, z\right), d\left(u_{n}, u_{n+1}\right), d(z, T z), \frac{d\left(u_{n}, T z\right)+d\left(z, u_{n+1}\right)}{2}\right\} \\
& =r d(z, T z) .
\end{aligned}
$$

Hence, as $r<1$, we obtain $d(z, T z)=0$. Since $T z$ is closed, $z \in T z$.

Hence, we have shown that $z \in T z$ in all cases, which completes the proof. $\square$

Remark. The Theorem 2.1 provides the answer to the Question 1 posed in [12].

Corollary 2.1. Let $(X, d)$ be a complete metric space and $T$ be a mapping from $X$ into $C B(X)$.

Assume that there exists $r \in[0,1)$ such that $\phi(r) d(x, T x) \leq d(x, y)$ implies

$$
H(T x, T y) \leq r \max \{d(x, y), d(x, T x), d(y, T y)\}
$$

for all $x, y \in X$, where the function $\phi$ is defined as in Theorem 2.1. Then, there exists $z \in X$ such that $z \in T z$. 
Proof. It comes from Theorem 2.1 since (9) implies (3). $\square$

The Corollary 2.1 is the multivalued mapping generalization of the Theorem 2.2 of Kikkawa and Suzuki [3], and therefore of the Kannan fixed point theorem [4] for generalized Kannan mappings. Also, it is the generalization of the Theorem 2.1 of Damjanović and Đorić [13].

From the Corollary 2.1, we obtain an another corollary:

Corollary 2.2. Let $(X, d)$ be a complete metric space and $T$ be a mapping from $X$ into $C B(X)$.

Let $\alpha \in[0,1 / 3)$ and $r=3 \alpha$. Suppose that there exists $r \in[0,1)$ such that

$$
\varphi(r) d(x, T x) \leq d(x, y) \quad \text { implies } \quad H(T x, T y) \leq \alpha d(x, y)+\alpha d(x, T x)+\alpha d(y, T y)
$$

for all $x, y \in X$, where the function $\phi$ is defined as in Theorem 2.1. Then, there exists $z \in X$ such that $z \in T z$.

Considering $T$ as a single-valued mapping, we have the following result:

Corollary 2.3. Let $(X, d)$ be a complete metric space and $T$ be a mapping from $X$ into $X$. Suppose that there exists $r \in[0,1)$ such that

$$
\varphi(r) d(x, T x) \leq d(x, y)
$$

implies

$$
d(T x, T y) \leq r \cdot \max \left\{d(x, y), d(x, T x), d(y, T y), \frac{d(x, T y)+d(y, T x)}{2}\right\}
$$

for all $x, y \in X$, where the function $\phi$ is defined as in Theorem 2.1. Then, there exists $z \in X$ such that $z=T z$.

Corollary 2.3 is the generalization fixed point theorem [4]. Corollary 2.3 also is the generalization of the Theorem 3.1 of Enjouji et al. [14], since by symmetry, the inequality (3.3) in [14] implies the inequality (1) in Theorem 1.1. Considering generalizations of the Theorem 1.2, Popescu [15] obtained the same result with different function $\phi$.

\section{An application}

The existence and uniqueness of solutions of functional equations and system of functional equations arising in dynamic programming have been studied by using various fixed point theorems (see $[12,16,17]$ and the references therein). In this article, we will prove the existence and uniqueness of a solution for a class of functional equations using Corollary 2.3.

Throughout this section, we assume that $U$ and $V$ are Banach spaces, $W \subset U, D \subset V$ and $\mathbb{R}$ is the field of real numbers. Let $B(W)$ denote the set of all the bounded realvalued functions on $W$. It is well known that $B(W)$ endowed with the metric

$$
d_{B}(h, k)=\sup _{x \in W}|h(x)-k(x)|, \quad h, k \in B(W)
$$

is a complete metric space.

According to Bellman and Lee [18], the basic form of the functional equation of dynamic programming is given as

$$
p(x)=\sup _{y} H(x, y, p(\tau(x, y)))
$$


where $x$ and $y$ represent the state and decision vectors, respectively, $\tau: W \times D \rightarrow W$ represents the transformation of the process and $p(x)$ represents the optimal return function with initial state $x$. In this section, we will study the existence and uniqueness of a solution of the following functional equation:

$$
p(x)=\sup _{y}[g(x, y)+G(x, y, p(\tau(x, y))), \quad x \in W
$$

where $g: W \times D \rightarrow \mathbb{R}$ and $G: W \times D \rightarrow \mathbb{R} \rightarrow \mathbb{R}$ are bounded functions.

Let a function $\phi$ be defined as in Theorem 2.1 and the mapping $T$ be defined by

$$
T(h(x))=\sup _{y \in D}\{g(x, y)+G(x, y, h(\tau(x, y))\}, \quad h \in B(W), x \in W .
$$

Theorem 3.1. Suppose that there exists $r \in[0,1)$ such that for every $(x, y) \in W \times D$, $h, k \in B(W)$ and $t \in W$, the inequality

$$
\varphi(r) d_{B}(T(h), h) \leq d_{B}(h, k)
$$

implies

$$
|G(x, y, h(t))-G(x, y, k(t))| \leq r \cdot M(h(t), k(t)),
$$

where

$$
\begin{gathered}
M(h(t), k(t))=\max \{|h(t)-k(t)|,|h(t)-T(h(t))|,|k(t)-T(k(t))|, \\
\left.\frac{|h(t)-T(k(t))|+|k(t)-T(h(t))|}{2}\right\} .
\end{gathered}
$$

Then, the functional equation (11) has a unique bounded solution in $B(W)$.

Proof. Note that $T$ is self-map of $B(W)$ and that $\left(B(W), d_{B}\right)$ is a complete metric space, where $d_{B}$ is the metric defined by (10). Let $\lambda$ be an arbitrary positive real number, and $h_{1}, h_{2} \in B(W)$. For $x \in W$, we choose $y_{1}, y_{2} \in D$ so that

$$
\begin{aligned}
& T\left(h_{1}(x)\right)<g\left(x, y_{1}\right)+G\left(x, y_{1}, h_{1}\left(\tau_{1}\right)\right)+\lambda, \\
& T\left(h_{2}(x)\right)<g\left(x, y_{2}\right)+G\left(x, y_{2}, h_{2}\left(\tau_{2}\right)\right)+\lambda,
\end{aligned}
$$

where $\tau_{1}=\tau\left(x, y_{1}\right)$ and $\tau_{2}=\tau\left(x, y_{2}\right)$.

From the definition of mapping $T$ and equation (12), we have

$$
\begin{aligned}
& T\left(h_{1}(x)\right) \geq g\left(x, y_{2}\right)+G\left(x, y_{2}, h_{1}\left(\tau_{2}\right)\right), \\
& T\left(h_{2}(x)\right) \geq g\left(x, y_{1}\right)+G\left(x, y_{1}, h_{2}\left(\tau_{1}\right)\right) .
\end{aligned}
$$

If the inequality (13) holds, then from (14) and (17), we obtain

$$
\begin{aligned}
T\left(h_{1}(x)\right)-T\left(h_{2}(x)\right) & <G\left(x, y_{1}, h_{1}\left(\tau_{1}\right)\right)-G\left(x, y_{1}, h_{2}\left(\tau_{1}\right)\right)+\lambda \\
& \leq\left|G\left(x, y_{1}, h_{1}\left(\tau_{1}\right)\right)-G\left(x, y_{1}, h_{2}\left(\tau_{1}\right)\right)\right|+\lambda \\
& \leq r \cdot M\left(h_{1}(x), h_{2}(x)\right)+\lambda .
\end{aligned}
$$

Similarly, (15) and (16) imply

$$
T\left(h_{2}(x)\right)-T\left(h_{1}(x)\right) \leq r \cdot M\left(h_{1}(x), h_{2}(x)\right)+\lambda .
$$


Hence, from (18) and (19), we have

$$
\left|T\left(h_{1}(x)\right)-T\left(h_{2}(x)\right)\right| \leq r \cdot M\left(h_{1}(x), h_{2}(x)\right)+\lambda .
$$

Since the inequality (20) is true for any $x \in W$ and arbitrary $\lambda>0$, then

$$
\varphi(r) d_{B}\left(T\left(h_{1}\right), h_{1}\right) \leq d_{B}\left(h_{1}, h_{2}\right)
$$

implies

$$
\begin{gathered}
d_{B}\left(T\left(h_{1}\right), T\left(h_{2}\right)\right) \leq r \cdot \max \left\{d_{B}\left(h_{1}, h_{2}\right), d_{B}\left(h_{1}, T\left(h_{1}\right)\right), d_{B}\left(h_{2}, T\left(h_{2}\right)\right),\right. \\
\left.\frac{d_{B}\left(h_{1}, T\left(h_{2}\right)\right)+d_{B}\left(h_{2}, T\left(h_{1}\right)\right)}{2}\right\} .
\end{gathered}
$$

Therefore, all the conditions of Corollary 2.3 are met for the mapping $T$, and hence the functional equation (11) has a unique bounded solution.

\section{Authors' contributions}

Both authors equitably contributed draft text and the main results section. DĐ contributed the application section. Both authors read and approved the final manuscript.

\section{Competing interests}

The authors declare that they have no competing interests.

\section{Received: 14 January 2011 Accepted: 22 August 2011 Published: 22 August 2011}

\section{References}

1. Suzuki, T: A generalized Banach contraction principle that characterizes metric completeness. Proc Am Math Soc. 136, 1861-186 (2008)

2. Banach, S: Sur les opérations dans les ensembles abstraits et leur application aux équations intégrales. Fund Math. 3, 133-181 (1922)

3. Kikkawa, M, Suzuki, T: Some similarity between contractions and Kannan mappings. Fixed Point Theory Appl 8 (2008). Article ID 649749

4. Kannan, R: Some results on fixed points-II. Am Math Monthly. 76, 405-408 (1969). doi:10.2307/2316437

5. Nadler, SB Jr: Multi-valued contraction mappings. Pacific J Math. 30, 475-488 (1969)

6. Mizoguchi, N, Takahashi, W: Fixed point theorems for multi-valued mappings on complete metric spaces. J Math Anal Appl. 141, 177-188 (1989). doi:10.1016/0022-247X(89)90214-X

7. Daffer, PZ, Kaneko, H: Fixed points of generalized contractive multi-valued mappings. J Math Anal Appl. 192, 655-666 (1995). doi:10.1006/jmaa.1995.1194

8. Semenov, PV: Fixed points of multi-valued contractions. Funct Anal Appl. 36(2), 159-161 (2002). doi:10.1023/ A:1015682926496

9. Ćirić, LJ: Multi-valued nonlinear contraction mappings. Nonlinear Anal. 71, 2716-2723 (2009). doi:10.1016/j. na.2009.01.116

10. Ćirić, LJ: Fixed points for generalized multi-valued contractions. Matematički Vesnik. 9(24), 265-272 (1972)

11. Kikkawa, M, Suzuki, T: Three fixed point theorems for generalized contractions with constants in complete metric spaces. Nonlinear Anal. 69, 2942-2949 (2008). doi:10.1016/j.na.2007.08.064

12. Singh, SL, Mishra, SN: Coincidence theorems for certain classes of hybrid contractions. Fixed Point Theory Appl 2010, Article ID 898109 (2010). 14

13. Damjanović, B, Đorić, D: Multivalued generalisations of the Kannan fixed point theorem. FILO-MAT. 25, 125-131 (2011)

14. Enjouji, Y, Nakanishi, M, Suzuki, T: A Generalization of Kannan's fixed point theorem. Fixed Point Theory Appl 2009, Article ID 192872 (2009). 10

15. Popescu, O: Two fixed point theorems for generalized contractionswith constants in complete metric space. Central Eur J Math. 7(3), 529-538 (2009). doi:10.2478/s11533-009-0019-2

16. Liu, Z, Agarwal, RP, Kang, SM: On solvability of functional equations and system of functional equations arising in dynamic programming. J Math Anal Appl. 297, 111-130 (2004). doi:10.1016/j.jmaa.2004.04.049

17. Liu, Z, Wang, L, Kim, HK, Kang, SM: Common fixed point theorems for contractive type mappings and their applications in dynamic programming. Bull Korean Math Soc. 45(3), 573-585 (2008). doi:10.4134/BKMS.2008.45.3.573

18. Bellman, R, Lee, ES: Functional equations arising in dynamic programming. Aequationes Math. 17, 1-18 (1978). doi:10.1007/BF01818535 